\title{
The active magnetic tracking with scalable coverage: indoor navigation for smartphones
}

\author{
Michail Zhelamskij \\ Saint-Petersburg Polytechnical University Doctoral Department, Saint-Petersburg, 193168, Russia \\ Correspondence to: Michail Zhelamskij (zhelamsk@ rambler.ru)
}

Received: 16 March 2016 - Revised: 4 August 2016 - Accepted: 22 September 2016 - Published: 1 November 2016

\begin{abstract}
In this paper we explore how to use the magnetic tracking technique for indoor navigation. The six degrees of freedom (6 DoF) magnetic tracking technology is selected because it is less influenced by the environment and has the highest accuracy among other techniques. A new method is proposed, called the alternating direct current (ADC) method, which permits one to get a double update rate, reduce the influence of Earth's magnetic field by a factor of 2 and reduce the power consumption by a factor of 3 . The theoretical basis of the new magnetic tracking method is given, including a mathematical modeling of the local field, a mathematical model of the measurement of the positioning field by a 3-D transducer based on the Hall effect sensors, and the mathematical model for the estimation of the measurement errors. A new, net-like source of the positioning local magnetic field is proposed for indoor navigation with a scalable coverage area. A mathematical model of the distributed positioning field is given. The results of simulations are shown.
\end{abstract}

\section{Introduction}

The task of an indoor navigation system is to determine the position of a user, who is equipped with a small mobile receiver in closed spaces where the GNSS (Global Navigation Satellite Systems; a satellite-based navigation systems like GPS or GLONASS) signals are not directly available without assistance (White, 2015; Chao, 2013; Fuller and Malkos, 2014). We will consider here the most relevant application area of indoor navigation, the "mass market", where a static linear positioning error at a level of $\pm 1 \mathrm{~m}$ is achieved. Everyone has a smartphone, and by entering the large hall of a hypermarket, we will try to take advantage of modern technologies such as indoor navigation. However, modern smartphones, even equipped with GPS, currently do not allow one to achieve this.

The motivation of this work is determined by the following consideration. In order to be competitive, the system should fulfill not only a linear positioning but also the definition of the orientation of the mobile receiver. The positioning error should be similar to the size of the distinguishable objects but not worse than $\pm 0.1 \mathrm{~m}$. In closed rooms, such as the hall of a hypermarket, airports, or entertainment and sport halls, many people exclude direct visibility of every human. There- fore, the system of indoor navigation should be insensitive to the presence of human bodies. The technology should be implemented on the already existing gadgets - smartphones for example, and not require additional mobile equipment. The technology should not occupy the free space of a closed room with stationary equipment. This equipment must be hidden in walls, under the floor, behind the ceiling space. The stationary interior of closed rooms (partitions, walls, conductive and ferromagnetic elements) should not affect system performance. The system should not create its own electromagnetic interference and should be resistant to external interference.

In this work we will not consider dynamic positioning techniques, such as the pedestrian navigation - which takes place while walking around an urban environment (IPIN, 2015). Also the mechanical positioning technology (the tactile system) is excluded from our consideration. Besides, we will not consider the "high sensitive GNSS", which due to strong signal attenuation provides accuracy at a level of only $\pm 10 \mathrm{~m}$, which is not enough for our task. The pseudosatellites (pseudolites), like the GNSS repeaters, will also not be considered here because of the strong influence of interior. The inertial navigation system will not be reviewed because it is not suitable for stationary positioning. 
The main aim of this article is the investigation and analysis of the positioning capabilities of the new method that satisfies the above requirements for indoor navigation. The paper is organized as follows: first we evaluate the main parameters for the selected method of indoor navigation, such as the possible update rate and the specific power consumption. We also consider the influence of external factors such as the Earth's magnetic field and the surrounding medium. Further, the requirements for both mobile and fixed parts of the indoor navigation system will be examined. The investigation is completed with the mathematical and physical modeling of the movement of a user in the proposed system of indoor navigation with scalable coverage.

The technology of indoor navigation presented here is suitable for a modern smartphone that has a video, an audio, a magnetometer, and a radio channel. To illustrate the new technique, we will present here only the static positioning method, when the mobile receiver is motionless during the measurement. The extension to the dynamic positioning will be presented elsewhere.

\section{The literature review}

Consider, what features are provided with current navigation technology, and whether they can be applied to modern user gadgets, i.e., smartphones?

The reference (IPIN, 2015; Mautz, 2012) contains an overview of the following well-known indoor navigation technologies. Optical systems for visible light contain both the cameras on the mobile object, and the markers on the environment. In the case of the invisible infrared light the systems contain the active LED-badges on the users, and fixed receivers. A passive system is applied to detect natural human radiation. Optical scanning systems exist such as "Kinect" (Microsoft, 2016), polar systems, or laser trackers, acoustic positioning systems (sound, ultrasound) use the triangulation method, and there are known the radio frequency (RF) technologies, such as WiFi systems, RFID (radio frequency identification), time-of-arrival ultra wideband (UWB), active beacons, ZigBee, Bluetooth, or GSM (global system for mobile communication) fingerprinting. The ultra hybrid systems are using inertial navigation and dead reckoning. A priori mapping of magnetic fingerprints is also applied for navigation.

The main drawback of the known technologies is the influence of the surrounding environment, like the reflection from the walls and furniture. Moreover, the multipath, the influence of moving people and opening doors and the limitation of positioning in one room due to the signal attenuation when passing obstacles with high density (walls, floors, ceilings) determine additional difficulties of known technologies. All the above methods have a need for direct visibility. Only linear coordinates are being calculated and the orientation cannot be determined. The optical methods need in- formation about the distance in order to convert the image to coordinates. The triangulation systems have a large size. Most of the technologies can not be implemented on the basis of existing smartphones.

In review (IPIN, 2015; Mautz, 2012) it is shown that all abovementioned technologies are very sensitive to the environment and require a free line of sight. As a result, the accuracy of the positioning is poor, at a level not better than $\pm(1-2) \mathrm{m}$. Therefore, only the technologies suitable for the smartphone shown in Table 1 are compared and analyzed. The data from references (Mandal Al, 2005; Navizon, 2016; Dardari et al., 2012; Daly et al., 2010) are also summarized. To find the way to eliminate the drawbacks also a known system based on active magnetic positioning at local fields (Melzer and Moffit, 1997; Hansen, 1986; Blood, 1997) is also added to the consideration.

Table 1 shows that the magnetic systems have an accuracy of 3 orders of magnitude better than all other technologies. Furthermore, the six degrees of freedom (6 DoF) magnetic tracking technology is less influenced by the environment. This basic magnetic technology includes creating a local magnetic field with a known spatial distribution. The accurate measurement of the field components is provided with a small mobile receiver. Therefore, a comparison of the measured values with the calculated fields for the initially approximated coordinates is being carried out, and the iterative approaching of coordinates from initial approximation to actual values allows one to obtain the desired output data.

To find the six coordinates, the system must provide at least six independent measurements at each point of the positioning zone. That is why the classical magnetic tracker includes three orthogonal windings ( $W=3$ ) with common centers, combined in one fixed source of local magnetic field, and three orthogonal sensors $(S=3)$ in the mobile receiver. In this case, nine independent measurements are being provided $W \times S>6$. This configuration is taken as a prototype for the task. Disadvantages of the prototype are described below. The main advantage of this technology is that it can be implemented on the basis of the modern smartphone, which has a 3-D magnetometer for measuring the Earth's magnetic field (EMF).

The known active magnetic trackers are based on AC (alternating current with sinusoidal shape - Fig. 1; Hansen, 1986) or DC (direct current with pulse shape - Fig. 2; Blood, 1997; Altman et al., 2009; Scully and Schneider, 2015) local magnetic field. In the first case (AC), all windings of the source are working simultaneously $\left(I_{x}+I_{y}+I_{z}\right)$ on different frequencies. In the second case they are operating in the pulse mode, one by one, sequentially $I_{x} \rightarrow I_{y} \rightarrow I_{z}$. The Fig. 2 shows that all pulses are unipolar here. The last graph on this figure is highly schematically. Actually, the rise and fall of pulse current is a much shorter than the pulse duration. The pause $\left(t_{3}-t_{4}\right)$ is designed to take into account the EMF, the influence of which is subtracted from each of the measured signals, obtained from the windings. 
Table 1. An evaluation of basic parameters of known technologies of indoor navigation, which are most suitable for modern smartphones.

\begin{tabular}{lllll}
\hline Technology & $\begin{array}{l}\text { Max. } \\
\text { accuracy } \\
{[\mathrm{m}]}\end{array}$ & $\begin{array}{l}\text { Max. } \\
\text { coverage } \\
{[\mathrm{m}]}\end{array}$ & $\begin{array}{l}\text { Power } \\
\text { consumption* }\end{array}$ & References \\
\hline Video cameras & $\sim 0.1$ & $\sim 10$ & autonomous & \\
Sound & $\sim 0.1$ & $\sim 10$ & $>1 \mathrm{wt} \mathrm{m}^{-1}$ & Fuller and Malkos (2014) \\
WLAN/WiFi & $\sim 1$ & $<50$ & $>1 \mathrm{wt} \mathrm{m}^{-2}$ & IPIN (2015) \\
Passive RFID & $\sim 1$ & $<50$ & $>2 \mathrm{wt} \mathrm{m}^{-2}$ & Microsoft (2016) \\
Ultra wideband & $\sim 1$ & $<50$ & $>1 \mathrm{wt} \mathrm{m}^{-2}$ & Mautz (2012) \\
Magnetic systems & $<0.01$ & $<4$ & $>6.25 \mathrm{wt} \mathrm{m}^{-2}$ & $\begin{array}{l}\text { Mandal Al et al. (2005); } \\
\text { Navizon (2016); } \\
\end{array}$ \\
& & & & Dardari et al. (2012) \\
\hline
\end{tabular}

* the estimations of the power consumption in Table 1 were taken for external equipment only (if required), not for the smartphone itself. Power consumption is given in specific units of $\left(\mathrm{W} \mathrm{m}^{-2}\right)$ - watts of power per meter in square- unit of the positioning zone area. The spacing between nodes affects accuracy. Therefore, the maximum accuracy does not correspond to maximum coverage. For all methods the calculation was made for $5 \mathrm{~m}$ spacing, selected to estimate the result accuracy.

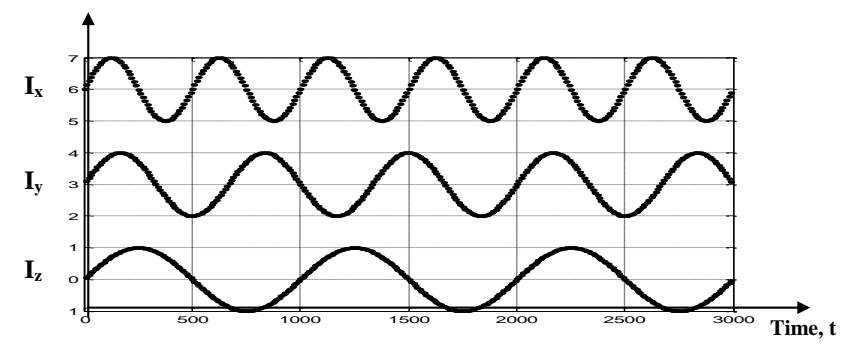

Figure 1. Dimensionless charts of the excitation currents through the windings of the sinusoidal magnetic positioning systems.

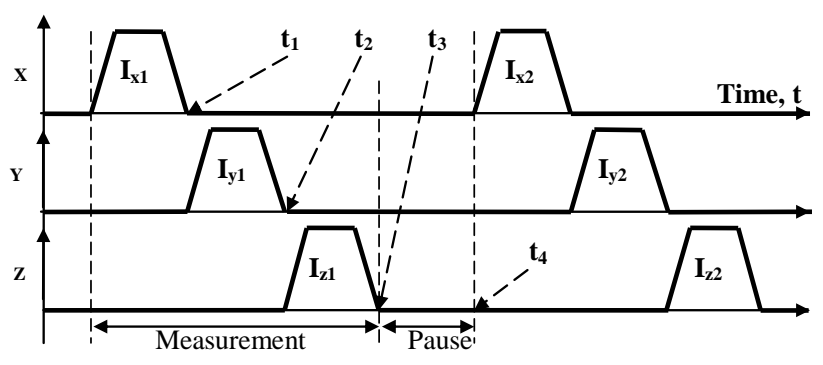

Figure 2. Dimensionless charts of the excitation currents in pulse magnetic positioning systems.

In both cases at each point of the trajectory nine measurements are performed by three sensors from three windings. Then, at each point the system of nonlinear equations is solved using iterative approximation methods, based on the initial approximation of coordinates.

The achievable parameters of a modern $6 \mathrm{DoF}$ magnetic DC tracker include the operation distance - up to 1 or $3 \mathrm{~m}$ depending on the source size; accuracy - about $\pm 1 \mathrm{~mm}$, and \pm 0.5 angle degree for $1 \mathrm{~m}$ distance; the update frequency up to $100 \mathrm{~Hz}$; environmental influence - both magnetic; and electrically conductive materials for $\mathrm{AC}$ - method and only magnetic for DC. The magnitude of induction of the local positioning magnetic field is at the level of EMF.

The influence of motionless ferromagnetic elements is eliminated by the mapping (Jones, 2002) of the magnetic field over the coverage area. The mapping is carried out by a priori sequential measurement of induction vectors from each winding at each grid node, covering the whole of the positioning area. That is why the moving ferromagnetics should not be situated inside the zone of movement. Due to the measurements performed on the plateau of the pulse, the effect of electro conductive elements is determined by the ratio between magnetic field pulse duration and eddy current decay time constant, exited in these elements by a pulse magnetic field. For a DC tracker the contribution of EMF depends on the object velocity and for a stationary object is zero. The dielectric environment elements have no effect (unlike all the alternative methods of indoor navigation) on the positioning accuracy due to full transparency of the magnetic field.

There have been several trials to apply the active magnetic positioning to the indoor navigation task (Pirkl and Lukowicz, 2013). The author of this paper tried to apply a resonance kind of AC method for tracking humans. As a result the accuracy of linear positioning (ca. $60 \mathrm{~cm}$ ) was demonstrated at the estimated distance no more than $1.5 \mathrm{~m}$. Unfortunately there is no indication of how the authors are going to increase the coverage area above the standard $1 \mathrm{~m}$. Moreover, the proposed inductive sensors are absent in the standard modern mobile communication devices. In addition, such sensors have low noise immunity, due to the large area of their measurement coils, and this trial does not successfully solve the indoor navigation problem. Therefore, we will further consider only the DC method. To evaluate the possibility of using magnetic tracking in indoor navigation, we will define the most visible disadvantages of magnetic technology. 


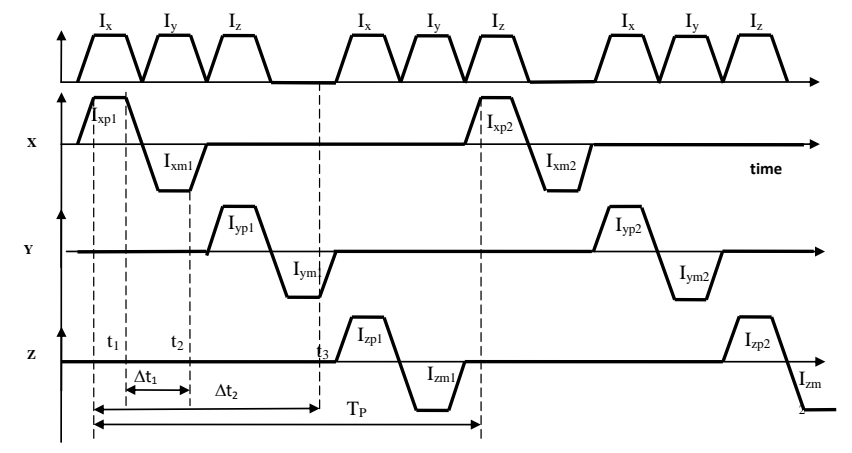

Figure 3. Schematically comparison of ADC and DC methods to organize the field of positioning.

\section{The task definition for indoor navigation with magnetic tracking}

The analysis shows that the main problems and drawbacks of known magnetic tracking for the indoor navigation are based on a limited tracking area. The positioning zone of $1 \mathrm{~m}$ is not enough for use in large halls of supermarkets and airports. The power consumption is also very significant - about $100 \mathrm{~W}$ over a sphere with a $4 \mathrm{~m}$ radius. It is important to take into account the need of the EMF and to provide the positioning of the moving object, not just the fixed objects. A limited update frequency has taken place - the magnetic fields switches up to 4 times faster than the output is updated, as shown on Fig. 2. This means that in order to further increase the output frequency, it is necessary to reduce the duration of the magnetic field pulses, which will increase the effect of eddy currents in the interior.

That is why it can be seen that the existing magnetic tracking technology does not fully meet the requirements of the indoor navigation. Therefore, this paper describes the results of an investigation of the possibility to improve the magnetic trackers for indoor navigation. The refined requirements for the developed magnetic system of indoor navigation shall be based on increased accuracy of positioning, which must be at the level of the abovementioned possibilities of active magnetic tracking for a local, limited coverage area. The coverage area in this case must be unlimited or, at least, scalable - in order to have the ability to increase the area by adding hardware. The infrastructure required for the operation should be of a minimum. The external equipment of an indoor navigation system should not extend or restrict the free space of application halls. To extend the functionality, the output data should contain six coordinates instead of three like the other abovementioned technologies. For the convenience of the users update rate should be no worse than $1 \mathrm{~Hz}$ in the mode of indoor navigation. The way to increase the update rate must be determined to provide the tracking of movable objects. Regarding the number of users - it should have a dual mode - single, or multiple users. For example, in the sport application the single user mode is important to train of athletes. In hypermarkets and airports multiple users mode is more in demand. The system latency of output data is indifferent for the static measurements. The method must provide dynamic measurements in the long run more for sport applications. For a mass market and other similar applications, the use of static measurements seems reasonable.

As can be seen from this analysis - none of the above technologies, including known magnetic tracking, allows one to get closer to the requirements listed above. This proves the relevance of establishing of a system of indoor navigation based on magnetic tracking, with an extended coverage area, minimized power of consumption, and reduced influence of EMF.

Next, let us look at ways to address the abovementioned issues.

\section{Proposal of a new method for organizing of the positioning field}

To satisfy the requirements, formulated above regarding accuracy, coverage area, infrastructure, number of coordinate, update rate, number of user and influence of EMF, a new method called the alternating direct current (ADC) method is proposed (Zhelamskij, 2011). The main idea of this new method is its special way of managing the local magnetic positioning field. Unlike the unipolar DC method, a new ADC method proposes bipolar current pulse to excite the same windings. Figure 3 shows the comparative chart of pulse currents in the source windings for a DC prototype (top graph, as the sum of the currents through all three windings for the same source like on Fig. 2), and for the new ADC method (three lower graphics, separately for each of windings). In both cases the graphics are presented for three orthogonal windings of local positioning systems (Hansen, 1986; Blood, 1997). Each winding contains about a hundred turns with full current around $700 \mathrm{amps}$. The source on a distance of $1 \mathrm{~m}$ has a shape of a cube with a side of $100 \mathrm{~mm}$. The magnetic field pulses duration and amplitudes are equal for both methods for this figure. As above, the rise and fall of pulse current is much shorter than the pulse duration. It is seen in the top graph that for the DC method the current pulses are separated by a pause, as shown in detail on Fig. 2. In the new method the bipolar current pulses are continuously supplied, running consecutively one by one without pause. Let us consider now the main features of new method.

At the top of each bipolar impulse the positioning field vector is folded with the EMF at a mobile receiver:

$\boldsymbol{B}_{m}=\boldsymbol{B}_{\mathrm{EMF}}+\boldsymbol{B}_{\mathrm{P}}$,

where $\boldsymbol{B}_{m}-$ is the total induction vector, measured by the sensors of the mobile receiver, $\boldsymbol{B}_{\mathrm{EMF}}-$ is the vector of EMF, and $\boldsymbol{B}_{\mathrm{P}}-$ is the vector of positioning field. 


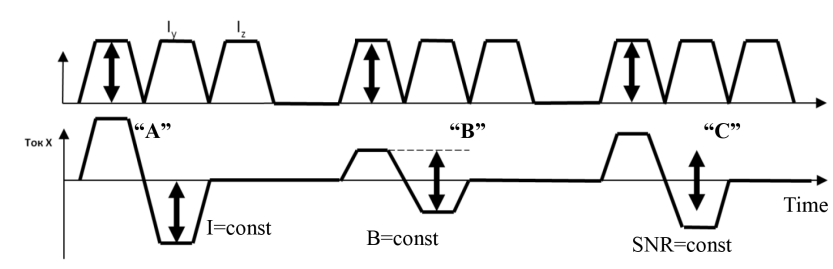

Figure 4. Three modes of ADC method operation.

Using Eq. (1) the system of equations for the two time moments $t_{1}$ and $t_{2}$ separated by an interval $\Delta t_{1}$ as shown in Fig. 3 are

$$
\left\{\begin{array}{l}
\boldsymbol{B}_{\mathrm{EMF}}\left(t_{1}\right)+\boldsymbol{B}_{\mathrm{P}}\left(t_{1}\right)=\boldsymbol{B}_{m}\left(t_{1}\right) \\
\boldsymbol{B}_{\mathrm{EMF}}\left(t_{2}\right)-\boldsymbol{B}_{\mathrm{P}}\left(t_{2}\right)=\boldsymbol{B}_{m}\left(t_{2}\right)
\end{array}\right.
$$

This system (Eq. 2) can be solved relative to the vector of the positioning field $\boldsymbol{B}_{\mathrm{P}}$. Subtracting the parts of the above equation from each other, and assuming equality of currents through the source winding $|I(+)|=|I(-)|$ at the moments $t_{1}$ and $t_{2}$, the common solution of the system (Eq. 2) is

$B_{\text {meas }}=2 \boldsymbol{B}_{m}=\left[\boldsymbol{B}_{\mathrm{P}}\left(t_{1}\right)+\boldsymbol{B}_{\mathrm{P}}\left(t_{2}\right)\right]+\left[\boldsymbol{B}_{\mathrm{EMF}}\left(t_{1}\right)-\boldsymbol{B}_{\mathrm{EMF}}\left(t_{2}\right)\right]$.

where $B_{\text {meas }}$ - full measured value of positioning field induction, which is applied to calculate the coordinates.

The residual value of the EMF, $\Delta B_{\mathrm{EMZ}}=$ $\left[\boldsymbol{B}_{\mathrm{EMF}}\left(t_{1}\right)-\boldsymbol{B}_{\mathrm{EMF}}\left(t_{2}\right)\right]=f(\omega, \Delta t)$, from Eq. (3) depends on both the angular velocity $\omega$ of the object, and the time interval $\Delta t_{1}=t_{2}-t_{1}$. The value $\Delta B_{\mathrm{EMZ}}$ is zero for the stationary object, when $\omega=0$.

Hence, taking into account that $\Delta t_{2} \geq 2 \Delta t_{1}$, the use of a bipolar positioning field allows for doubling the amplitude of measured induction vs. DC method, and twice reducing the impact of EMF:

$B_{\text {meas }}=2 B_{\mathrm{P}}, \Delta B_{\mathrm{EMFADC}} \leq \frac{1}{2} \Delta B_{\mathrm{EMFDC}}$,

where $\triangle B_{\mathrm{EMFADC}}$ - effect of EMF for new ADC - method; $\Delta B_{\mathrm{EMFDC}}$ - effect of EMF for DC - prototype.

Additionally, as follows from Fig. 3 , the condition $\Delta t_{2} \geq$ $2 \Delta t_{1}$ from Eq. (4) means a 2 -fold increase of update rate vs. the original, reference DC method. Then consider the ratio of the averaged power consumption for the DC- method $\left(P_{1}\right)$, and the ADC-method $\left(\mathrm{P}_{2}\right)$, which is

$$
\frac{P_{1}}{P_{2}}=\frac{\frac{1}{t_{\mathrm{P}}} \int_{0}^{t_{\mathrm{P}}=4 T} I_{x}^{2} R \mathrm{~d} t}{\frac{1}{t_{\mathrm{P}}} \int_{0}^{t_{P}} I_{x p m}^{6 T} R \mathrm{~d} t},
$$

where $R$ is the full resistance of winding and $T$ the duration of one unipolar pulse of positioning field, which is equal for both DC and ADC methods.
Three possible modes of the ADC method operation are illustrated on Fig. 4. From Eq. (5) the results show that if the amplitudes of the pulsed current are really equal $I_{x}=I_{x p}=$ $I_{x m}$ (mode " $A$ " on Fig. 4 ) we get $P_{1} / P_{2}=3 / 4$; i.e., the DC method is more economical in this mode because of the current pauses existence. If the sweep of measured inductions are equal $B_{\mathrm{DC}}=2 B_{\mathrm{ADC}}$ (mode " $B$ "), we get $P_{1} / P_{2}=3$; i.e., the ADC method is 3 times more economical than a prototype in this mode of the same value of the measured induction. If the RMS (root mean square) of noise is given $\sigma_{N}=$ constant, then the signal-to-noise ratio for the prototype $\mathrm{SNR} 1=B_{1} / \sigma_{N}$, and $\mathrm{SNR}_{2}=1.41 \times B_{2} / \sigma_{N}$ for the bipolar fields method are identical $\mathrm{SNR}_{1}=\mathrm{SNR}_{2}$ (mode " $C$ ") for twice reduced power consumption $P_{1} / P_{2}=2$, as seen in the following calculations:

$\frac{B_{1}}{\sigma_{N}}=\frac{2 \cdot B_{2}}{\sigma_{N} \cdot \sqrt{2}}, I_{2}=\frac{\sqrt{2}}{2} I_{1}, P_{2}=\frac{1}{2} \cdot P_{1}$,

where $B_{1}$ and $B_{2}$ are positioning field pulse amplitudes in DC and ADC methods, respectively, at which the condition $\mathrm{SNR} 1=\mathrm{SNR} 2$ is fulfilled.

A full parameter comparison of the DC and ADC methods is shown in the Table 2. The update rate and the magnitude of measured induction are doubled in accordance with Eq. (4). The module of the induction vector at a great distance from the windings with current can be described by the following simplified formula:

$B \approx \Psi / R^{3}$

where $\Psi$ is the function that describes the effect of the sizes of the winding, and the flowing current. In accordance with Fig. 3, for the same currents the module values used to calculate the coordinates for the prototype $B_{1}$ and for the new method $B_{2}$, which differ by half, will read as follows: $B_{1}=$ $\frac{\Psi}{R_{1}^{3}}, B_{2}=\frac{2 \Psi}{R_{2}^{3}}$. Equating $B_{1}=B_{2}$, we get $\left(R_{2} / R_{1}\right)^{3}=2$, or $R_{2}=1.25 \times R_{1}$. Therefore, Table 2 shows that the new proposed ADC method provides an increased positioning zone radius of about $25 \%$. The impact of EMF is reduced at least twice in accordance with Eq. (4). It follows from Eq. (5) that the power consumption is reduced by up to a factor of 3 . A slight increase in latency can be offset by the application of a Kalman filter, performing a "forward extrapolation".

Due to the presented advantages, it is worth taking a look at a new way of organizing for the positioning field, because it better meets the conditions for indoor navigation for the update rate, power consumption, and the impact of EMF.

Now, having an improved method for magnetic tracking, one needs to understand how to use this proposal for addressing indoor navigation. 
Table 2. Comparison of parameters of the prototype and new method.

\begin{tabular}{lll}
\hline Parameter & DC & ADC \\
\hline Update frequency for same pulse & $F$ & $2 F$ \\
duration & & \\
Measured inductance deviation & $\Delta B$ & $2 \times \Delta B$ \\
Coverage zone radius & $R$ & $1.25 \times R$ \\
Latency & 4 & 6 \\
Power consumption & $P$ & $1.25 P(I=$ Const $)$ \\
& & $0.33 P(\Delta B=$ Const $)$ \\
Earth field influence & & $0.5 P(\mathrm{SNR}=$ Const $)$ \\
& 4 & 2
\end{tabular}

\section{The new approach of positioning local field creation and measurement}

In addition to Zhelamskij (2014a, b), the theoretical basis of the new magnetic tracking method for the indoor navigation is given here in more detail, including a mathematical model of the positioning field, a mathematical model of the measurement of the positioning field with a 3-D Hall effect sensors, and a mathematical model of the measurement errors.

\subsection{Mathematical model of positioning field}

The expression for the projection of the induction vector, created at the observation point with the coordinates of $x_{m}, y_{m}$, $z_{m}$, by one of the positioning field source winding, on the normal surface to the sensor surface of the mobile receiver with the following orientation angles: $\alpha$-azimuth, $\beta$ - elevation and $\gamma$-roll, as shown in Fig. 5, is written as

$$
\begin{gathered}
B_{m}\left(x_{m}, y_{m}, z_{m}, \alpha, \beta, \gamma, t\right)=\Phi(X, M)=F_{1}\left(x_{m}, y_{m}, z_{m}\right) \\
\cdot F_{2}(\alpha, \beta, \gamma) \cdot F_{3}(t),
\end{gathered}
$$

where $F_{1}\left(x_{m}, y_{m}, z_{m}\right)=\left[B_{x}\left(x_{m}, y_{m}, z_{m}, A, B, I\right), B_{y}\left(x_{m}\right.\right.$, $\left.\left.y_{m}, z_{m}, A, B, I\right), B_{z}\left(x_{m}, y_{m}, z_{m}, A, B, I\right)\right]^{T}$ is the function that defines the dependence of the axial components of induction vector on the linear coordinates; $A, B$ are the side length of the rectangular winding; $F_{2}(\alpha, \beta, \gamma)=S_{1 r} \times A_{x y z}$ is the function describing the sensor orientation influence on the measured value; $A_{x y z} \equiv A_{x}(\alpha) \times A_{y}(\beta) \times A_{z}(\gamma)$ is full rotation matrix on three angle in the sequence $\alpha \rightarrow \beta \rightarrow \gamma$; $A_{x}(\alpha), A_{y}(\beta), A_{z}(\gamma)$ are three matrix with the size $3 \times 3$, reflecting the coordinate transformation for the sequential object rotation around each axis of the stationary 3-D Euclidean space $X Y Z . F_{3}(\mathrm{t})$ is the function that specifies the sequence of the independent fields over time, as illustrated in Fig. 3, respectively, for the winding $X \rightarrow Y \rightarrow Z$ :

$$
F_{3 X}(t)=\left\{\begin{array}{c}
+1 \\
-1 \\
0
\end{array}\right\} \text { for }\left\{\begin{array}{l}
0<t<T \\
T<t<2 T \\
2 T<t<6 T
\end{array}\right.
$$

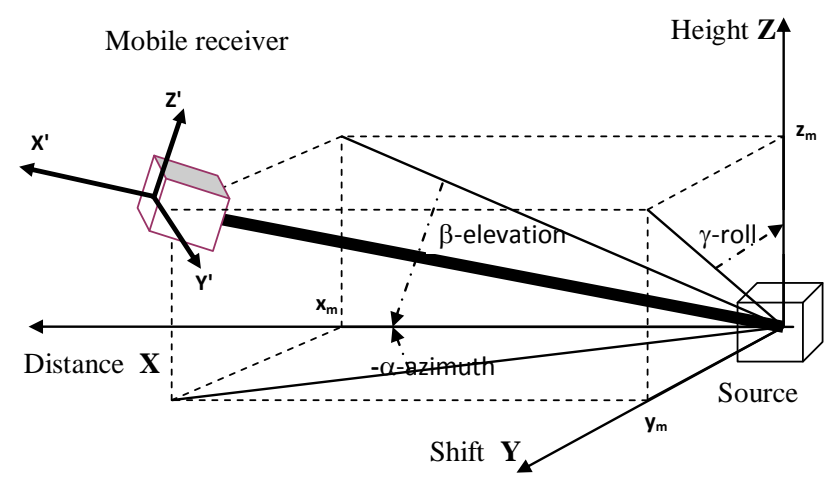

Figure 5. Linear position and orientation of mobile receiver relative to the source in Cartesian coordinate system.

$$
\begin{aligned}
& F_{3 Y}(t)=\left\{\begin{array}{l}
0 \\
+1 \\
-1
\end{array}\right\} \text { for }\left\{\begin{array}{l}
0<t<2 T, 4 T<t<6 T \\
2 T<t<3 T \\
3 T<t<4 T
\end{array}\right. \\
& F_{3 Z}(t)=\left\{\begin{array}{l}
0 \\
+1 \\
-1
\end{array}\right\} \text { for }\left\{\begin{array}{l}
0<t<4 T \\
4 T<t<5 T, \\
5 T<t<6 T
\end{array}\right.
\end{aligned}
$$

where $T$ is the half period of bipolar pulse $T=\Delta t_{1}=t_{2}-t_{1}$ on Fig. 3. It is evident from Eq. (7) that the measured value $B_{m}$ is represented as the product of three functions with independent arguments - linear coordinates, angular orientation, and time. That is why the model, given in Eq. (7), allows one to explore a positioning field at different conditions, as the shape and size of windings, their mutual location, and orientation. The effect of the orientation of the mobile receiver will be investigated. The frequency of the winding switching is to be optimized. The transients duration and the influence of electrically conductive and ferromagnetic materials on the tracking accuracy are very important to research. The result of the simulation will allow one to verify the requirements for the elements of the distributed source. The accuracy of the mathematical description of the positioning field will be investigated below.

Furthermore, the positioning field is not only important for indoor navigation but also measuring technology, which is considered below.

\subsection{Mathematical model of the positioning field measurement by the 3-D Hall effect sensors}

Let us consider a 3-D magnetometer based on the Hall effect sensors, which is available in modern smartphones, as a mobile receiver for the positioning field. These magnetometers are intended for measurement of homogeneous Earth's magnetic field only. However, the local positioning field is not uniform. Therefore, the mathematical model is proposed to apply this magnetometer to measure the inhomogeneous positioning field (Zhelamskij, 2014a). The model is written 
as follows:

$$
\begin{aligned}
U_{\text {out }}(t) & =\left\{K _ { M } \cdot \left[B_{\mathrm{MT}}(x, y, z, \alpha, \beta, \gamma, t)+\Delta B_{\mathrm{EMF}(t)}\right.\right. \\
& \left.\left.+\Delta B_{F(x, y, z)}\right] \cdot\left(1+\sum_{k=1}^{8} \varepsilon_{s k}(x, y, z)\right)\right\} \\
& +U_{\text {shift }(t, T)}+U_{\mathrm{RAND}(t)},
\end{aligned}
$$

where $K_{M} \approx G(f) \times \operatorname{MS}(T=$ Const $\pm \Delta T) \times I_{\mathrm{HG}} \times I_{S}$ is the function, which takes into account the sensitivity of the sensor MS, an amplification $G(f)$ of the measuring channel, and the excitation currents both through the sensors $I_{\mathrm{HG}}$ and field source $I_{S} ; B_{\mathrm{MT}}=F_{1}\left(x_{m}, y_{m}, z_{m}\right) \times F_{2}(\alpha, \beta, \gamma) \times F_{3}(t)$ is the projection of the induction vector of the positioning field from Eq. (7), intended to measure; $\sum_{k=1}^{8} \varepsilon_{s k}(x, y, z)$ is the total systematic measurement error, where $\varepsilon_{S 1}$ is the effect of spatial diversity of the receiver sensors, $\varepsilon_{S 2}$ is the effect of temperature, $\varepsilon_{S 3}$ is mutual non-orthogonality of sensors, $\varepsilon_{S 4}$ is the effect of sensors excitation current, $\varepsilon_{S 5}$ is the effect of source excitation current, $\varepsilon_{S 6}$ is sensitivity uncertainty, $\varepsilon_{S 7}$ is error of analog-digital converter, $\varepsilon_{S 8}$ is the influence of sensors finite size. $\Delta B_{\mathrm{EMF}}(\mathrm{t})$ is the residual impact of EMF; $\Delta B_{F}(x, y, z)$ is the accuracy of the mathematical description of the positioning field; $U_{\text {shift }}(t, T=$ Const $\pm \Delta T)$ is the offset of sensor output voltage; $U_{\mathrm{RAND}}(t)$ is a random process, modeling its own noise of measurement device.

It can be seen from Eq. (8) that the total induction applied to the sensor:

$$
B_{\Sigma}=B_{\mathrm{MT}}(x, y, z, \alpha, \beta, \gamma, t)+\Delta B_{\mathrm{EMF}}(t)+\Delta B_{F}(x, y, z),
$$

is converted to the measured voltage through the function $K_{M}$ with an accuracy of $\sum_{k=1}^{8} \varepsilon_{s k}(x, y, z)$. Then voltages $U_{\text {shift }}(t, T)$ and $U_{\text {RAND }}(t)$ are added to measured voltage $U_{\text {out }}$, which do not depend on the measured fields induction. Thus, the model of the measured value includes the induction of a local magnetic field, full set of systematic measurement errors, and randomized noise.

As a result, the mathematical model for the systematic positioning error of the field measurement is given by

$$
P(D)=\frac{\Delta B_{x}}{B_{x}}(D)=\sum_{1}^{8} \varepsilon_{s k}+\varepsilon_{\mathrm{EMF}}(D)=P_{1}(D)+P_{2}
$$

where $P_{1}(D)=\varepsilon_{s 1}(D)+\varepsilon_{s 7}(D)+\varepsilon_{s 8}(D)+\varepsilon_{\mathrm{EMF}}(D) ; P_{2}=$ $\varepsilon_{s 3}+\varepsilon_{s 2}+\varepsilon_{s 4}+\varepsilon_{s 5}+\varepsilon_{s 6} \neq f(D), D$ is the distance between receiver and source.

The specificity of the positioning field measurements results from Eq. (9), where the full error depends not only on the magnitude, but also on the distance $(D)$. It can be seen from Eq. (9) that some components depend on the distance, like $P_{1}(D)$, whereas others do not, such as $P_{2}$. This fact allows one to consider the expression in Eq. (9) as a function of the distance $P(D)$, which has an extremum. Minimizing $P(D)$ was performed by the dichotomy method under the following condition:

$\frac{\mathrm{d} P(D)}{\mathrm{d} D} \rightarrow 0$.

It is shown below that the condition in Eq. (10) can be solved at different distances $(D)$, depending on the properties of the measuring system. The investigations have shown that is possible to build a positioning field measurement system, where a minimum measurement error will be obtained at a given distance between the source and receiver.

The presented mathematical model of measurements of positioning field allows for starting the creation of an indoor navigation theory. This theory must explore the impact of the properties of the receiver and analog-digital converters into positioning accuracy. The influence of distance and the errors of mathematical description of the positioning field should also be explained. The investigation of this model made it possible to formulate the requirements for mobile receiver for the different task's condition. The first consideration of the metrological conditions of indoor navigation, based on the proposed model of measurement, are fulfilled in Zhelamskij (2015), where the models and tools for both measurement and reproducing inhomogeneous positioning magnetic fields are offered.

Now let us try to link the proposed mathematical models into a common indoor navigation system based on magnetic tracking.

\section{The theory of new indoor navigation based on the magnetic tracking}

\subsection{Mathematical model of distributed positioning fields}

A new, net-like source of the positioning local magnetic field is proposed in Zhelamskij (2014a) for the indoor navigation with a scalable coverage area. The fragment of the distributed source is shown on Fig. 6. The power supply is not shown here and will be considered elsewhere. The windings, marked with the same numbers, are working simultaneously in bipolar pulse mode. The user with a moveable smartphone receiver is designated by the star.

A mathematical model of the distributed positioning field for the windings of the group " 1 " will be written as follows:

$B_{m}\left(x_{m}, y_{m}\right)=\sum_{k=0}^{K} \sum_{n=0}^{N} \mathrm{FM}\left(x_{m}-k \cdot 2 S, y_{m}-n \cdot 2 S\right)$,

where $B_{m}\left(x_{m}, y_{m}\right)$ is the value of the induction vector module, measured by the mobile receiver at the point with coordinates $x_{m}, y_{m}$; FM is the mathematical model of induction vector module from Eq. (7), created by one winding of distributed source; $S$ is the step of distributed source that defines the distance between the nearest windings in the line; 
$k, n$ are the numbers of winding in one group on the $x$ and $y$ axes, respectively; int $K=X_{\max } / S$, int $N=Y_{\max } / S$ are the total number of windings on the axes; $x_{m}, y_{m}$ are the currently user coordinates relative distributed source. $t$ is seen from Eq. (11) that measured induction is the sum of the contributions from all the windings of distributed source.

Based on the above considerations a formula is proposed that links the mutual removal of windings $S$ with their magnetic moment $M$, the number of measurements $N$ to be averaged, and the minimum measured induction $B_{\min }$ to get a sustainable tracking within frame of one square:

$\frac{B_{\min }}{\sqrt{N}}=\mu \mu_{0} \cdot \frac{M}{(2 \cdot 1.41 \cdot S)^{3}}$.

If the condition in Eq. (12) is satisfied, it results from Eq. (7) that $\operatorname{FM}\left(x_{m}-k \cdot 2 S, y_{m}-n \cdot 2 S\right) \rightarrow 0$ for $\left(k \neq x_{m} / S, n \neq\right.$ $\left.y_{m} / S\right)$.

This means that in this case, the main contribution to the measured value of the induction vector module at any point of positioning area is created by the winding nearest to the user. The influence of other windings is thus negligible. In this case the expression in Eq. (11) coincides with the expression in Eq. (7) within one square of the grid:

$$
\begin{gathered}
B_{m}\left(x_{m}, y_{m}, z_{m}, \alpha, \beta, \gamma, t\right)=F_{1}\left[\left(x_{m}, y_{m}, z_{m}\right) \in R_{n, k}\right] \\
\cdot F_{2}(\alpha, \beta, \gamma) \cdot F_{3}(t) .
\end{gathered}
$$

Therefore, the components of the induction vector included in factor $F_{1}$ of Eq. (7) are recorded as follows for flat rectangular coils, which have a magnetic moment oriented vertically:

$$
\begin{aligned}
B_{\mathrm{ZZ}}= & \mu \mu_{0} \cdot I_{3} \cdot\left(\frac{E_{4} \cdot\left(\frac{E_{2}}{F_{2}}+\frac{E_{1}}{F_{1}}\right)}{z_{m}^{2}+E_{4}^{2}}+\frac{E_{2} \cdot\left(\frac{E_{3}}{F_{4}}+\frac{E_{4}}{F_{2}}\right)}{z_{m}^{2}+E_{2}^{2}}\right. \\
& \left.+\frac{E_{3} \cdot\left(\frac{E_{1}}{F_{3}}+\frac{E_{2}}{F_{4}}\right)}{z_{m}^{2}+E_{3}^{2}}+\frac{E_{1} \cdot\left(\frac{E_{3}}{F_{3}}+\frac{E_{4}}{F_{1}}\right)}{z_{m}^{2}+E_{1}^{2}}\right), \\
B_{Y Z} & =\mu \mu_{0} \cdot I_{z} \cdot\left(\frac{z_{m} \cdot\left(\frac{E_{3}}{F_{4}}+\frac{E_{4}}{F_{2}}\right)}{z_{m}^{2}+E_{2}^{2}}+\frac{z_{m} \cdot\left(\frac{E_{3}}{F_{3}}+\frac{E_{4}}{F_{1}}\right)}{z_{m}^{2}+E_{1}^{2}}\right), \\
B_{X Z} & =\mu \mu_{0} \cdot I_{z} \cdot\left(\frac{z_{m} \cdot\left(\frac{E_{1}}{F_{1}}+\frac{E_{2}}{F_{2}}\right)}{z_{m}^{2}+E_{4}^{2}}+\frac{z_{m} \cdot\left(\frac{E_{1}}{F_{3}}+\frac{E_{2}}{F_{4}}\right)}{z_{m}^{2}+E_{3}^{2}}\right),
\end{aligned}
$$

where $B_{X Z}, B_{Y Z}, B_{Z Z}$ are the values of the axial component of induction vector, generated at the observation point by the coil $Z ; E_{1}=E+y_{m} ; E_{2}=E-y_{m} ; E_{3}=F-x_{m} ; E_{4}=$ $F+x_{m} ; F_{1}=\sqrt{z_{m}^{2}+E_{1}^{2}+E_{4}^{2}} ; F_{2}=\sqrt{z_{m}^{2}+E_{2}^{2}+E_{4}^{2}} ; F_{3}=$ $\sqrt{z_{m}^{2}+E_{1}^{2}+E_{3}^{2}} ; F_{4}=\sqrt{z_{m}^{2}+E_{2}^{2}+E_{3}^{2}}, E=A, F=B$ are half of the winding $Z$ sides on axis $y$ and $x$ respectively; $I_{z}$ is the full current through the winding; $z_{m}$ is the height of the

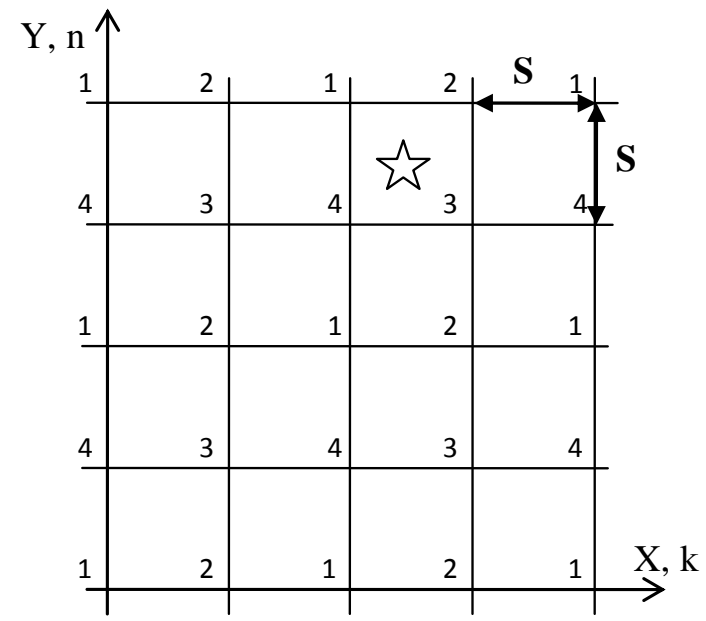

Figure 6. A location of windings in the nodes of the net - like the distributed source of positioning field.

observation point above the winding; $\mu$ is the relative magnetic permeability of the medium; $\mu_{0}=4 \pi \times 10^{-7} \mathrm{H} / \mathrm{M}$ is the absolute magnetic permeability.

Cofactor $F_{2}(\alpha, \beta, \gamma)$ retains its appearance as in Eq. (7), and the third factor $F_{3}(t)$ is determined by the time interval scale $T_{\mathrm{P}}=6 T$ (see Fig. 3) included in this expression, which in this case should be selected, taking into account the requirements for the update frequency $f_{\mathrm{U}}=1 /\left(T_{\mathrm{P}} / 3\right)<1 \mathrm{~Hz}$. As a result, due to the selection of the parameters in accordance with Eq. (12), the same mathematical model for the magnetic fields of the distributed source is being used, as it was for the local source Eq. (7) described above.

The windings of the distributed source have a flat shape and can be located in the interior elements - in walls, ceilings, or basically under the floor. The numerical simulation shown (Zhelamskij, 2015) that both orientation of source windings are available as horizontal and vertical. The former can be situated in the floor or the ceiling of the room. The vertical oriented windings can be located behind the walls, not occupying the free space of room. Simultaneously it was considered that the flat winding round shape, rectangular, or freeform agreed with the building designs of the room. The mathematical models of magnetic field are given in Zhelamskij (2015) for all the mentioned shape of windings and their orientations.

The operation of distributed source has a number of features, which are discussed below.

\subsection{A synchronization between autonomous mobile receiver and distributed source}

An additional tracking task for a distributed magnetic field is how one synchronizes the user mobile receiver with the operation sequence of a distributed source. A method of binding the receiver's mobile software to the sequence of switching 


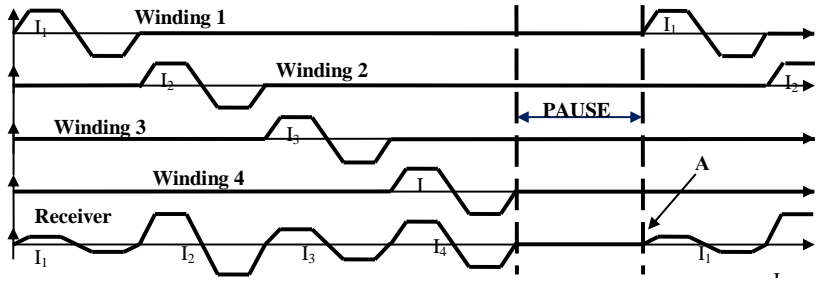

Figure 7. The synchronization pause to start the calculation procedure at autonomous receiver.

groups of the distributed source windings (sync in time) is proposed for autonomous user, who has no connection with the source. The point of synchronization is the introduction of additional breaks into a sequence of the bipolar pulses, as shown in Fig. 7.

The pause is introduced periodically, such as once over $30 \mathrm{~s}$, for example. The duration of the pause must be enough for the recognition by the autonomous mobile receiver, for example $T \approx 1 \mathrm{~s}$. After a pause, the system is operating continuously, until the next break. The top four charts show the sequence of the bipolar currents through the winding groups "1-4" as shown above in Fig. 6. The bottom graph shows the signal from one of the sensors of the mobile receiver, which combines the fields from all windings.

It can be seen that the pause confidently allows for detecting when the distributed source starts at moment " $A$ ". The receiver recognizes the signal of winding $1,2,3$, and 4 because the order of the windings switching is predefined and immutable. The first signal after a pause is from winding 1 , next - from winding 2 , third - from winding 3 , and finally, the last one is from winding 4 . This sequence is maintained throughout the work source. Besides, the receiver knows the pulse duration $T$, and that each couple of pulses is symmetrical, as follows from bottom curve of Fig. 7. These three conditions (winding sequence $1 \rightarrow 2 \rightarrow 3 \rightarrow 4, T=$ Const, $I_{+}=I_{-}$), together with the pause existing, are providing the basement of the recognition algorithm. Thus, the proposed method of synchronization allows one to use a stand-alone receiver that has no direct connection with the distributed source.

\subsection{The initial capture of the coordinates}

It is equally important to understand the start of positioning; i.e., in which square of the distributed source a user is situated when the smartphone could be switched on at anytime.

The initial binding of mobile receiver in the coordinate system of the distributed source is a one-time procedure, which is carried out everywhere over the coverage zone at any time at the user's request to initialize the positioning calculation. A method for the initial capture of coordinates is proposed here, based on an additional excitation of reference sinusoidal oscillations over the individual frequencies in the distributed windings.

We assign each winding its own frequency, value of which is defined by the expression:

$f_{n, k}=f_{n}+\Delta f \cdot k$,

where $f_{n}$ is the start frequency of line, and $\Delta f$ is the frequency deviation between neighboring columns.

It is supposed further that each winding creates a magnetic field at the assigned frequency all the time, excepting the bipolar positioning field duration, as shown in Fig. 8. In this case, the mobile receiver will see the following signal:

$B_{m}=\sum_{k=0}^{K} \sum_{n=0}^{N} \operatorname{FM}\left(x_{m}-k \cdot S, y_{m}-n \cdot S\right) \cdot \sin \left(2 \pi f_{n, k}\right)$

Taking into account above mathematical model of the distributed positioning field, expression Eq. (14) looks like this:

$$
\begin{aligned}
& B_{m}=\mathrm{FM}\left(x_{m}-k \cdot S, y_{m}-n \cdot S\right) \cdot \sin \left(2 \pi f_{n, k}\right) \\
& +\mathrm{FM}\left(x_{m}-(k+1) \cdot S, y_{m}-n \cdot S\right) \cdot \sin \left(2 \pi f_{n+1, k}\right)+ \\
& +\mathrm{FM}\left(x_{m}-(k+1) \cdot S, y_{m}-(n+1) \cdot S\right) \cdot \sin \left(2 \pi f_{n+1, k+1}\right) \\
& +\mathrm{FM}\left(x_{m}-k \cdot S, y_{m}-(n+1) \cdot S\right) \cdot \sin \left(2 \pi f_{n, k+1}\right) .
\end{aligned}
$$

Hence, sinusoidal signals will be registered only from the nearest windings, while the rest will attenuate due to remoteness. Next, a discrete Fourier transformation for the full signal Eq. (15) is performed, adopted by the mobile receiver for a full cycle of distributed source excitation

$$
\begin{aligned}
& \operatorname{Re} X[k]=\sum_{i=0}^{N-1} x[i] \cdot \cos (2 \pi k i / N), \\
& \operatorname{Im} X[k]=\sum_{i=0}^{N-1} x[i] \cdot \sin (2 \pi k i / N), \\
& X[k]=\sqrt{\operatorname{Re}^{2}+\operatorname{Im}^{2}}
\end{aligned}
$$

In view of the fact that

$$
\begin{aligned}
& \operatorname{FM}\left(x_{m}-k \cdot S, y_{m}-n \cdot S\right) \neq 0, \\
& \operatorname{FM}\left(x_{m}-(k+1) \cdot S, y_{m}-n \cdot S\right) \neq 0, \\
& \operatorname{FM}\left(x_{m}-(k+1) \cdot S, y_{m}-(n+1) \cdot S\right) \neq 0, \\
& \operatorname{FM}\left(x_{m}-k \cdot S, y_{m}-(n+1) \cdot S\right) \neq 0,
\end{aligned}
$$

we will get from Eq. (15) all the frequencies of the sinusoidal oscillation, created by the windings, closest to the user: $f_{n, k}$, $f_{n+1, k}, f_{n+1, k+1}, f_{n, k+1}$.

It is obvious that to identify the square in the net it is enough to define its two peaks, located diagonally, as shown in Fig. 9 by circles.

Therefore, having a priori the binding frequency to the windings of the distributed source, it is possible to define a square grid in which the mobile receiver is placed, and to start 


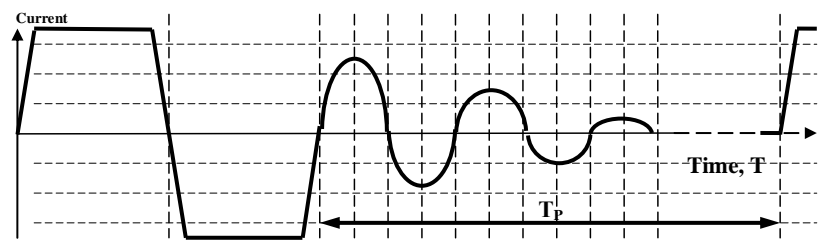

Figure 8. Dimensionless free oscillations of current in the winding after completion of bipolar pulse.

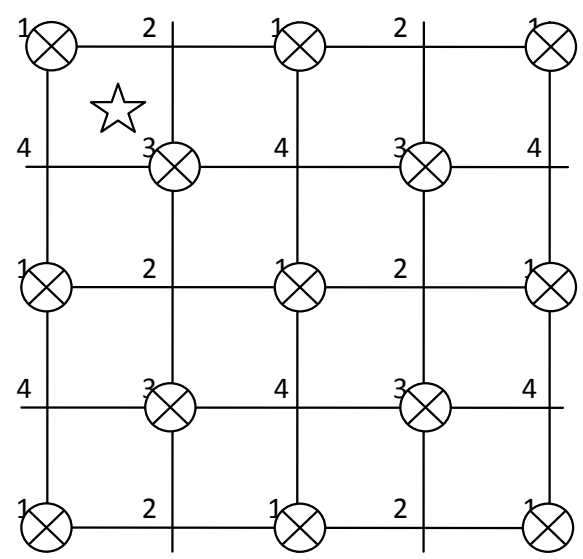

Figure 9. The location of winding with oscillating circuits to be used for an initial capture to start a positioning.

the positioning. Thus, the proposed method of initial capture of the mobile receiver coordinates allows to eliminate the disambiguate solutions of the linear positioning, associated with the periodic nature of the distributed fields.

\subsection{Effect of environment}

The new method has to be robust to changing environmental conditions.

There is no influence of non-metallic interior elements (walls, ceilings, floors) on the positioning accuracy as the magnetic permeability of these materials is equal to one $(\mu \rightarrow 1)$. Such materials do not affect the distribution of the magnetic field in the transfer zone. Influence of humans are also missing for the same reason, because the human body is transparent to the magnetic field. It is also not affected by the presence of electrically conductive non-magnetic elements, such as aluminum, titanium, or copper, since the duration of the magnetic field pulse is much larger than the eddy currents decay time constant in these elements. For example, the duration of the field rise - unit $\mathrm{ms}$, pulse duration $-1 \mathrm{~s}$, and the decay time constant in aluminum $3 \mathrm{~mm}$ thick - hundreds of microseconds.

In some cases, the stationary ferromagnetic iron may be situated within the zone of positioning, such as ferromagnetic racks or building structures. The influence of the iron can be reduced by the mapping of the positioning field gridded with steps agreed with the linear positioning error. In this case, for the distributed source in Fig. 6, the grid of mapping can be about $0.3 \mathrm{~m}$. Full vector of position field at an arbitrary point $B_{\mathrm{F}}$ is a sum vector from winding $B_{\mathrm{W}}$ plus vector of amendment $B_{\mathrm{A}}$ :

$\boldsymbol{B}_{\mathrm{F}}=\boldsymbol{B}_{\mathrm{W}}+\boldsymbol{B}_{\mathrm{A}}$

The procedure for obtaining of amendments is to advance (and periodic, if necessary) measuring the total field at the nodes of the mapping grid. Measured values of $B_{\mathrm{F}}$ are determined a priori for each node of mapping grid. The map of amendments is obtained from Eq. (17) also a priori as the difference between the measured value $B_{F}$ and computed by mathematical models (Eqs. 7, 13) value $B_{\mathrm{W}}$. The interpolation of the amendments from grid nodes to arbitrary coordinates of the point of observation is made in the real time during positioning.

Next we ask the question - what will be happen if the vehicle like tram or bus will pass near the positioning zone? The answer is - nothing will happen. First, in accordance with Eq. (6) the field falls as the cube of the distance. That is, at the distance (3-4) $\mathrm{m}$ from the source the induction of residual field is $<10^{-3} \%$ of the field in the center of the coil. The presence of any iron in such a low field has negligible effects on the rest field in the positioning zone. Second, it is necessary to watch that the new iron does not appear in the positioning area, and when it does appeared one needs to produce a repeated mapping.

\section{The results of numerical and physical simulations}

\subsection{An investigation of the operation mode for the magnetic indoor navigation}

The first numerical estimations were conducted for the hall of the hypermarket, where it is necessary to provide steady positioning of arbitrarily moving users. For this case the initial parameters of the distributed source of the positioning field are shown in Table 3 . The windings with square shape are arranged under the non-magnetic floor in the same plane. The height of the movable receiver is limited by the growth of humans and does not exceed $H=2 \mathrm{~m}$ from the source plane. Bipolar pulse duration is determined by the mode of operation of the mobile receiver and will be considered below. For the given parameters and without averaging of measurements $(N=1)$ the formula (12) allows to get the requested magnetic moment $M$ of winding at the level:

$M=\frac{B_{\min } \cdot(2.82 \cdot S)^{3}}{\mu \mu_{0}}=600 A \cdot \mathrm{m}^{2}$, with turns number $w=666$.

As specified above in Table 3, we get periodic distributed source fields, as shown in Fig. 10 for one group of windings 
Table 3. Main parameters of distributed source.

\begin{tabular}{ll}
\hline Parameter & Value \\
\hline Winding spacing $-S[\mathrm{~m}]$ & 3 \\
Side length of the rectangular winding: $A=B[\mathrm{~m}]$ & 0.3 \\
Excitation current of winding $-I$ [Amper] & 10 \\
Minimum field induction can be measured $B_{\min }[\mu T]$ & 10 \\
\hline
\end{tabular}

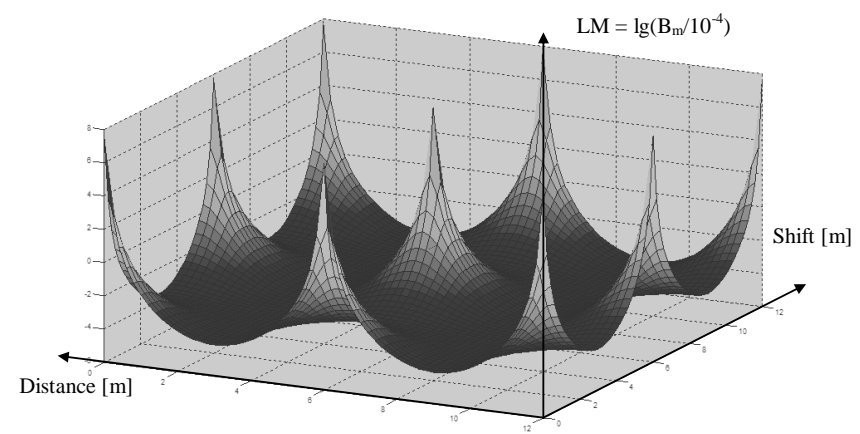

Figure 10. Periodic positioning field of distributed source.

"1", activated simultaneously at the same time. The number of such windings in the group " 1 " is equal to $N_{1} \times K_{1}=9$ from total number of $N \times K=25$. The module of the induction vector is plotted on the vertical axis in logarithmic scale. On the horizontal axis the offset and distance within the designated fragment is plotted, containing the overall number $5 \times 5$ windings, as shown in Fig. 6 . The coverage zone has an area of $A=144 \mathrm{~m}^{2}$ in this case.

Calculation was carried out for the height $H=0.5 \mathrm{~m}$ above the source plane. The recurrent nature of the field can be seen. The minimum induction in the valleys between the windings is about $10 \mu \mathrm{T}$. Maximum induction over the windings shall not exceed $2 \mathrm{mT}$. To provide an unambiguous connection between the magnetic field induction and coordinates, the initial capture of the coordinate is being applied to find the starting square of the distributed source where the user is situated. The elimination of the field symmetry influence will be discussed below.

\subsection{Movement simulation}

The numerical simulation of the linear movement was simulated on the fragment of distributed source as shown on Fig. 11. In this case, one can record the coordinates of the six windings: $1 \rightarrow\left[x_{1}, y_{1}\right], S 2 \rightarrow\left[x_{2}, y_{2}\right], S 3 \rightarrow\left[x_{3}, y_{3}\right]$, $S 4 \rightarrow\left[x_{4}, y_{4}\right], S 5 \rightarrow\left[x_{5}, y_{5}\right], S 6 \rightarrow\left[x_{6}, y_{6}\right]$. The direction of the receiver movement is indicated by the broken line from left to right.

Due to the obvious symmetry of the position field, shown in Fig. 10, a mobile receiver can not distinguish its position relative to the symmetry line shown in Fig. 10, for example in the field of winding $S 2$ or $S 5$. On both sides of this line of

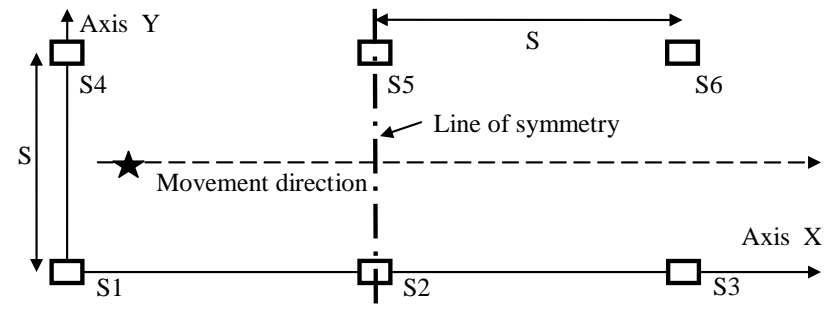

Figure 11. The object flow through the periodic elements of the distributed source.

symmetry it will show the same results of measurement that will make the mistake of coordinates calculation. In this case it is important to define the conditions of crossing the border of neighboring squares distributed source. Let us consider these conditions.

The linear coordinates of the mobile object $x, y, z$ can be found from the solution of the system of nonlinear equations, written in the form of six equations, describing the modules of the induction vectors at the mobile receiver, produced by each of the distributed windings:

$$
\left\{\begin{aligned}
B_{m 1 c}= & \sqrt{B_{x 1}^{2}+B_{y 1}^{2}+B_{z 1}^{2}} \\
B_{m 2 c}= & \sqrt{B_{x 2}^{2}+B_{y 2}^{2}+B_{z 2}^{2}} \\
--- & \sqrt{B_{x 6}^{2}+B_{y 6}^{2}+B_{z 6}^{2}}
\end{aligned}\right.
$$

where $B_{m 1 c}-B_{m 6 c}$ are the calculated values of the modules of the magnetic induction vectors, produced by the six winding of the distributed source, respectively at the point of the initial approximation of the coordinates using the analytical description of the positioning field Eq. (13); $B_{x 1}, B_{y 1}, B_{z 1}$, etc. - the values of the orthogonal projections of the field induction vector from the winding on the axis of the mobile system of coordinates, measured by the mobile receiver.

The functional $\mathrm{CF}$ is subject to minimization, compiled from the discrepancies of the six couple of measured and calculated modules of the induction vectors from different windings, as follows:

$$
\begin{aligned}
& \mathrm{CF}=\left(B_{m 1 m}-B_{m 1 c}\right)^{2}+\left(B_{m 2 m}-B_{m 2 c}\right)^{2}+\left(B_{m 3 m}-B_{m 3 c}\right)^{2}+ \\
& +\left(B_{m 4 m}-B_{m 4 c}\right)^{2}+\left(B_{m 5 m}-B_{m 5 c}\right)^{2}+\left(B_{m 6 m}-B_{m 6 c}\right)^{2} .
\end{aligned}
$$

All measured values are being obtained at one point of the transfer zone for a stationary receiver. The solution of the system Eq. (18) is obtained at each point of positioning zone by the steepest-descent method. The iteration formula in this case takes the following form:

$|x y z|_{k+1}^{T}=|x y z|_{k}^{T}-\operatorname{step}_{1} \cdot \operatorname{grad}|\mathrm{CF}|$,

where $|x y z|_{k+1}^{T}$ is the transposed matrix of the current linear coordinates; $|x y z|_{k}^{T}$ is the initial approximation of the coordinates; and $\operatorname{grad}[\mathrm{CF}]=[\partial \mathrm{CF} / \partial x, \partial \mathrm{CF} /, \partial y, \partial \mathrm{CF} / \partial z]^{T}$ is 
the transpose matrix of the spatial derivatives of the functional at the point of the initial approximation; step $_{1}-$ is the step of the iteration process, the rate of convergence of which is ensured by the choice of its value.

A cycle of windings excitation to be applied to the Fig. 11 fragment of distributed source will be described with the following sequence $(S 1+S 3) \rightarrow S 2 \rightarrow S 5 \rightarrow(S 4+S 6)$. The winding $S 1$ and $S 3$, and then $S 4$ and $S 6$ - are being excited simultaneously.

The exception of symmetry solution relative on line $S 2$ $S 5$ on Fig. 11 is made as follows. In the user's proximity to the square border $S 2-S 5$, the sign of the total induction vector $\left(B_{x}\right)$ is checked, according to the results of the induction measurements from the group of symmetrical windings $(S 1+S 3)$ and $(S 4+S 6)$. To do this, we must find the values of induction vector projections on the axis of the stationary coordinate system $B_{x}, B_{y}, B_{z}$, solving the following system of equations:

$\left\{\begin{array}{l}B_{x} \cdot \cos \varphi_{1 R}+B_{y} \cdot \cos \nu_{1 R}+B_{z} \cdot \cos \xi_{1 R}=B_{m 1} \\ B_{x} \cdot \cos \varphi_{2 R}+B_{y} \cdot \cos \nu_{2 R}+B_{z} \cdot \cos \xi_{2 R}=B_{m 2} \\ B_{x} \cdot \cos \varphi_{3 R}+B_{y} \cdot \cos \nu_{3 R}+B_{z} \cdot \cos \xi_{3 R}=B_{m 3}\end{array}\right.$.

Here $\cos \left(\phi_{1}\right)_{R}-\cos \left(\xi_{3}\right)_{R}$ are the cosines of guiding angles for the sensors in a fixed coordinate system, taken relative to the initial approximation; $B_{m 1}, B_{m 2}, B_{m 3}$ the value of magnetic inductions, measured with the orthogonal sensors. Such a transformation is intended to bring the orthogonal component of the induction vector, measured by a sensor with an arbitrary orientation, to the axial components of the fixed coordinate system, necessary for further analysis, comparison, and summation.

The same assessment is applied to find the direction of the induction vector, created by the windings $S 2$ and $S 5$. Performing the procedure in Eq. (20) twice, regarding the induction vectors created by windings $S 2$ and $S 5$, we obtain the sum of both projections of vectors on the $x$ axis: $B_{X}=$ $B_{X 2}+B_{X 5}$. Next, we accept that if $B_{X}=0$ than $x=x_{2}=x_{5}$, if $B_{X}<0$ than $x<x_{2}=x_{5}$, if $B_{X}>0$ than $x>x_{2}=x_{5}$.

In result, if $B_{X}<0$, then the calculation of the receiver coordinates is performed on mathematical field models for the following windings: $S 1\left(x_{1}, y_{1}\right), S 2\left(x_{2}, y_{2}\right), S 4\left(x_{4}, y_{4}\right), S 5\left(x_{5}\right.$, $y_{5}$ ). If the changes of component's directions $B_{X}>0$ is detected, then the program calculates the coordinates of the receiver on the other windings $S 2\left(x_{2}, y_{2}\right), S 3\left(x_{3}, y_{3}\right), S 5\left(x_{5}\right.$, $\left.y_{5}\right), S 6\left(x_{6}, y_{6}\right)$, which correspond to the intersection of lines of symmetry of the receiver. Thus, the proposed conversion of measured data allows for setting the membership of the current coordinates of the receiver $x_{m}, y_{m}, z_{m}$ to one or the other square of the distributed source.

A similar procedure is being applied when the user is approaching the borders $(S 1-S 2)$ or $(S 4-S 5)$. In this case, the sign of total components $B_{Y}$ is examined, instead $B_{X}$, like it was above.

The results of the numerical simulation of the movement are given in Zhelamskij (2014a), where the possibility of un- restricted movement is shown within the zone, covered by the distributed source, despite the symmetry and periodicity of the positioning field.

\subsection{DoF tracking at the scalable coverage area}

Based on the conditions of the task, six coordinates of the mobile object should be obtained during the positioning. Such an opportunity should be provided.

In addition to the linear positioning description (Zhelamskij, 2014b), the orientation angles of a mobile object in the case of a distributed source at given linear coordinates are found from the system of nonlinear equations of the following type

$$
\begin{aligned}
& F(x, y, z, \alpha, \beta, \gamma, \Psi)=B_{x} \cdot \cos x_{r}+B_{y} \cdot \cos y_{r} \\
& +B_{z} \cdot \cos z_{r}-B_{i}=0 .
\end{aligned}
$$

In the task of orientation one does not use the modular measurement, but the vector ones, where the orthogonal components of the induction vector of the positioning field are being measured. In this case, the system contains 12 equations in accordance with the number of independent measurements ( $N=3$ sensors in the magnetometer, and $K=4$ windings in each square distributed source):

$\boldsymbol{F}(X, \Psi)=\left[\begin{array}{c}F_{1}(x, y, z, \alpha, \beta, \gamma, \Psi) \\ \cdots \ldots \ldots \ldots \ldots \ldots \ldots \ldots \\ F_{12}(x, y, z, \alpha, \beta, \gamma, \Psi)\end{array}\right]=0$,

where $[x, y, z]=$ Const $\in R_{n, k}$ are linear coordinates, known from the solution of equations (Eq. 18), where $n, k$ are any integers, indicating the current number of windings in a distributed source.

A decision of system (Eq. 21) is based on a minimization of the following functionality:

$$
\mathrm{CF}=\mathrm{CF}=\sum_{n=1}^{N} \sum_{k=1}^{K}\left(\left(B_{k n c}(X)-B_{k n m}\right)^{2},\right.
$$

with the condition $N \times K=12$, which means 12 measurements, fulfilled by three sensors from four windings

For linear coordinates, obtained from the solution of system (Eq. 18), the steepest descent method is applied to minimize the functional (Eq. 22), with the iterative step selection by the method of dichotomy. For the angular coordinates it is described with the following iterative formula:

$$
|\alpha \beta \gamma|_{k+1}^{T}=|\alpha \beta \gamma|_{k}^{T}-\eta_{k} \cdot \nabla\left(\mathrm{CF}_{2 k},\right)
$$

where $\eta_{k}$ is the optimal step, defined from the dichotomy algorithm, implying a halving of the interval and the choice of the part, which leads to a decrease in functional (Zhelamskij, 2015).

The movable object orientation angles $[\alpha, \beta, \gamma]$ are a result of the convergence of an iterative process using Eq. (23). Figure 12 presents an illustration of the convergence of the iteration process for different values of initial orientations from which the calculation process starts. 


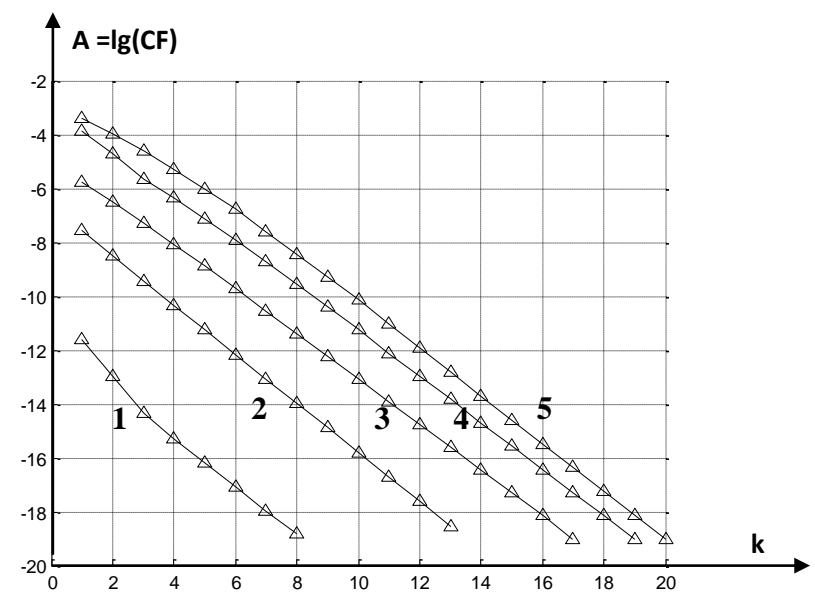

Figure 12. The dependence of functional CF (in logarithmic scale) vs. number of iterations " $k$ " in the convergence process for different initial starting approximations.

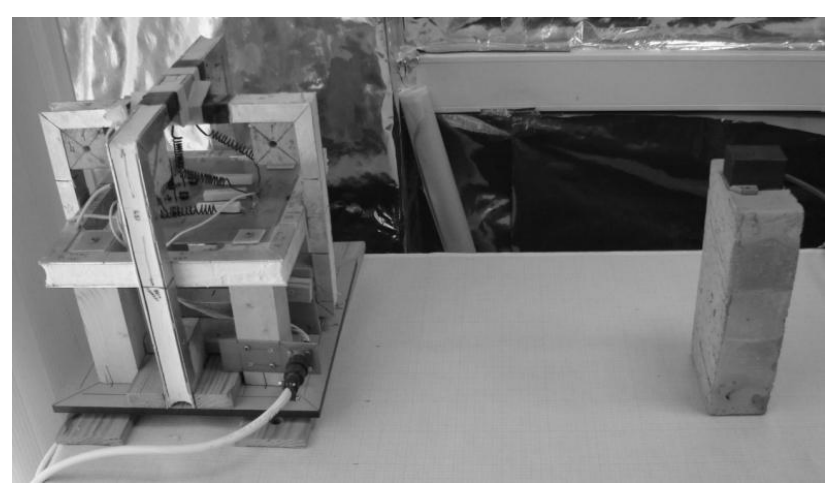

Figure 13. The initial position in the study of the ranges of magnetic tracking operation with concentrated source. On the left - a local source with orthogonal coils with common center, to the right - the movable receiver on the base.

The orientation of destination point is $[\alpha, \beta, \gamma]=[0.3$, $0.4,0.5]$ radian. The orientation of the initial approximation as starting point for the iteration process (from left to right on Fig. 12) is shown in Table 4.

As seen in Fig. 12 that the number of iterations " $k$ " depends on the elongation of initial approximation of orientation angles from the actually azimuth, elevation, and roll of object. The residual value of functionality $\mathrm{CF}$ after the final iteration is affected by the magnetic measurements errors. The data from Fig. 12 prove that the proposed method of indoor navigation, in addition to linear coordinates, allows for determining the orientation of a moving object, unlike the other methods mentioned above in Table 1 as prototype of indoor navigation. The ranges of the orientation angles are sufficient for the application in the task of mass market. Thus, these investigations show the ability to calculate the six coordinates of a mobile receiver at a fixed system of coordinate
Table 4. The orientation of initial approach.

\begin{tabular}{lccc}
\hline $\begin{array}{l}\text { Number of curve } \\
\text { on Fig. 12 }\end{array}$ & $\begin{array}{c}\alpha \\
{[\mathrm{Rad}]}\end{array}$ & $\begin{array}{c}\beta \\
{[\mathrm{Rad}]}\end{array}$ & $\begin{array}{c}\gamma \\
{[\mathrm{Rad}]}\end{array}$ \\
\hline 1 & 0.4 & 0.4 & 0.4 \\
2 & 0.5 & 0.5 & 0.5 \\
3 & 0.7 & 0.7 & 0.7 \\
4 & 1.2 & 1.2 & 1.2 \\
5 & 1.4 & 1.4 & 1.4 \\
\hline
\end{tabular}

of a source, instead of the three linear coordinates, described above.

\subsection{The basic experimental research: functional test}

At this initial stage, the handmade prototype to check the functioning of the main elements of the positioning system was set up as shown in Fig. 13. The prototype consist of - local source of position field (to the left), the movable receiver (to the right), and the computer (not shown). The windings in the source have a rectangular shape as described above for distributed source. The windings are arranged orthogonally to each other and have a common center. Dimensions of maximum winding are $0.28 \times 0.28 \mathrm{~m}$. Full current is $I_{\mathrm{FULL}}=630$ Amps. Number of turns is $w=105$. The duration of current ramp up $-\Delta t=400 \mathrm{mks}$. Inductance of winding $L=5 \mathrm{mHn}$. Power consumption in continuous mode $P_{\mathrm{C}}=43 \mathrm{~W}$. Weight of one winding $-W=2.1 \mathrm{kG}$. The amplitude of the positioning field induction at a distance of $1.5 \mathrm{~m}-\mathrm{B} \sim 0.1 \Gamma c$.

The composition of the mobile receiver includes three orthogonal Hall sensor, analog preamplifiers, which are the source of excitation current for sensors. The size of the sensors sensitive area is $100 \times 100 \mu \mathrm{m}$; the sensitivity is $100 \mathrm{mkV} \mathrm{Gs}^{-1}$; the resolution is $0.1 \mathrm{mGs}$; bandwidth is $300 \mathrm{~Hz}$; the volume of the receiver assembly is $27 \mathrm{sm}^{3}$; and power consumption is $2.8 \mathrm{~W}$. The thermal stabilization of sensors is being performed at level $\sim 70{ }^{\circ} \mathrm{C}$. Length of the connection cable to the computer is $10 \mathrm{~m}$.

The calculation the receiver coordinates is performed by iterative formulas $(19,23)$. These investigations have shown that the range of the orientation angles of movable object is $\Delta \phi= \pm 180^{\circ}$ each angle (azimuth, elevation and roll). The distance range of operation is $3 \mathrm{~m}$. Positioning accuracy in the first stage - not worse $\pm 1 \mathrm{sm}, \pm 5^{\circ}$. Stability of computed coordinates is not worse $\sigma_{X}<1 \mathrm{~mm}, \sigma_{\phi}<0.2^{\circ}$. The results of positioning along the direct and circular trajectories are given in Zhelamskij (2009, 2015). Similar results were obtained with three coils, which are placed horizontally in one common plane uniformly over the circumference with a diameter of $3 \mathrm{~m}$. 


\section{Discussion}

\subsection{Smartphone as a mobile receiver}

The magnetometers of the modern smartphones, designed to measure the EMF, are built mostly on the Hall effect sensors. The magnetometer AK8975 (Asahi Kasei Corp.) (Asahi - Kasei, 2016), with a size of about $3 \times 3 \times 1 \mathrm{~mm}$, contains three orthogonal Hall effect sensor, providing a 13 bit resolution or $\Delta B=0.3 \mu T$ on the scale $B_{\mathrm{MAX}}= \pm 1200 \mu T$. The similar magnetometer MPU 9150 (Invensense, 2016) from another manufacturer, contains AK8975 with the same parameters and dimensions. His modification MPU9250 provides a resolution of 16 bits already in the range $\pm 4800 \mu T$ with an auxiliary master bus. The magnetometer BMX055 (Bosh, 2016) is already a hybrid structure that contains two fluxgate transducer $x y$ and Hall effect sensor on $z$ axis. The resolution is not worse than $0.3 \mu T$ and the dimensions are $3 \times 4.5 \times 1 \mathrm{~mm}$. Therefore, we considered, for example, the product AK8975 as a prototype of a possible mobile receiver of positioning field at the smartphone.

In this case, the measuring time is $t_{\mathrm{m}}=10 \mathrm{~ms}$, on each of the three available channels. A full cycle of the measurement of the three orthogonal components of induction vector to calculate the module will take $T_{\mathrm{M}}=30 \mathrm{~ms}$. For reliable recognition of the pulsed positioning field by an autonomous smartphone, the duration of one module measurement $T_{\mathrm{M}}$ should be much less than the bipolar pulsed magnetic positioning field duration $T_{\mathrm{M}} \ll T_{\mathrm{P}}$. It is accepted for exactness that $T_{\mathrm{P}}=30 \times T_{\mathrm{M}}=1 \mathrm{~s}$. In this case the number of measurements for half of the bipolar pulse is $N=T_{\mathrm{P}} / 2 \times T_{\mathrm{M}}=15$, which will lead to a reduction of the average random error of factor $N^{1 / 2}=4$. The complete switching duration cycle for four groups of windings is $4 \mathrm{~s}$. Update rate is $-1 \mathrm{~Hz}$ and the latency is $-4 \mathrm{~s}$. Thus, if the fields pulse duration is specified, existing smartphones allows to perform a stationary object positioning, when $X=[x, y, z]=$ Const, already at this stage.

A variable orientation of the smartphone $\Phi=[\alpha, \beta, \gamma]=$ Var does not influence the linear positioning results. However, the obtained value of the field pulse duration would restrict the speed of linear motion of the smartphone during positioning. To increase the speed of the motion it is necessary to improve the frequency of measurements for a magnetometer, built into the smartphone. The update rate of $100 \mathrm{~Hz}$ will be reached with bipolar field pulse duration on level $T_{\mathrm{P}} \approx 10 \mathrm{~ms}$. In this case, for reliable recognition of the field pulses, the required sampling frequency of the measurements should be $f \geq 2 \mathrm{kHz}$, which would eliminate the restrictions on the movement of the smartphone. These magnetometers for smartphones are already arriving and they can count on the next R\&D stage of work.

In Table 5 we present the results of the numerical simulation for the error components of the measurement, which are mostly caused with the mobile receiver properties (Asahi -
Table 5. Content of error of magnetic induction field measurement.

\begin{tabular}{lll}
\hline Source of error & $D=0.3 \mathrm{~m}$ & $D=3 \mathrm{~m}$ \\
\hline$\varepsilon_{s 1}$ & $\leq \pm 0.1 \%$ & $\rightarrow 0$ \\
$\varepsilon_{s 2}$ & $\leq \pm 0.1 \%$ & $\ll$ \\
$\varepsilon_{s 3}$ & $\leq \pm 0.2 \%$ & $\ll$ \\
$\varepsilon_{s}$ & $\leq \pm 0.3 \%$ & $\ll$ \\
$\varepsilon_{s 5}$ & $\leq \pm 0.1 \% ;$ & $\ll$ \\
$\varepsilon_{s 6}$ & $\leq \pm 0.3 \%$ & $\ll$ \\
$\varepsilon_{s 7}$ & $\leq \pm 0.05 \%$ & $\leq \pm 0.35 \%$ \\
$\varepsilon_{s 8}$ & $\leq \pm 1 \%$ & $\rightarrow 0$ \\
\hline
\end{tabular}

Kasei, 2016). In addition, as shown below in Sect. 8.4, measurement errors are also determined by the nature of the positioning field. The simulation was made in accordance with the mathematical model of Eq. (8) for the positioning field measurement. The calculations were performed for two distances between the winding and receiver, and for a constant temperature of the environment.

The noise of the receiver is neglected $\varepsilon_{\text {noise }} \rightarrow 0$ for the selected field pulse duration. The size of the sensors does not affect the measurement accuracy $\varepsilon_{s} \rightarrow 0$, only at large distances from the source $D \rightarrow 3 \mathrm{~m}$. The influence of the spatial allocation of the sensors $\varepsilon_{s 1} \neq 0$ is only significant near the source, where $D \rightarrow 0.3 \mathrm{~m}$. The here described positioning system has high immunity to external interference due to low area of sensitive zone of each sensor in mobile receiver. The distributed source does not create its own interferences for another equipment due to a low rate of the magnetic field charging. In this case the condition in Eq. (10) $\frac{\mathrm{d} P(D)}{\mathrm{d} D} \rightarrow 0$ gives the following solution about overall error of measurement:

$D=\operatorname{Arg} \min \left\{\frac{\mathrm{d} P(D)}{\mathrm{d} D} \rightarrow 0\right\} \approx 1.5 \mathrm{~m}$.

So, the investigations shown that, for the considered layout, the systematic error of measurement has a minimum at the distance $D \sim 1.5 \mathrm{~m}$ between the winding and receiver.

\subsection{The duration of initial capture}

For a distributed source, as described in Sect. 7.1, one should evaluated the possible duration of the initial procedure to bind an autonomous receiver in the source coordinate system. As can be seen from Fig. 8, the break between bipolar pulses of the current in each winding, intended for initial binding, contains $T_{\mathrm{P}}=3 \mathrm{~s}$. In this case, the duration of bipolar pulse itself is $T_{\Pi}=1 \mathrm{~s}$. The initial binding sinusoidal signal is measured as the module of induction vector, using the signals from the three orthogonal sensors of the magnetometer. The value of the full signal module is accumulated for all four operational cycles of the distributed source to get all surrounding frequencies. In accordance with Fig. 6, we are considering the space with the area $144 \mathrm{~m}^{2}$, where the 25 
Table 6. Identification frequencies $[\mathrm{Hz}]$ at multi-level distributed source.

\begin{tabular}{cccccc}
\hline & $\begin{array}{c}1 \\
\text { column }\end{array}$ & $\begin{array}{c}2 \\
\text { column }\end{array}$ & $\begin{array}{c}3 \\
\text { column }\end{array}$ & $\begin{array}{c}4 \\
\text { column }\end{array}$ & $\begin{array}{c}5 \\
\text { colomn }\end{array}$ \\
\hline 1 row & 3 & - & 5 & - & 7 \\
2 row & - & 8 & - & 9 & - \\
3 row & 10 & - & 11 & - & 12 \\
4 row & - & 13 & - & 14 & - \\
5 row & 15 & - & 16 & - & 17 \\
\hline
\end{tabular}

winding are situated. The mathematical modeling shows that just two sinusoids on each square are enough for a reliable recognition. Table 6 shows an example of the distribution of initial binding sinusoidal signal frequencies among windings of distributed source.

The simulation showed that the duration of the initial capture of the coordinates is defined with only a time of the calculations of the signals spectrum in accordance with Eqs. $(15,16)$, which are observable at the point where the receiver situates. So, the initial capture may be performed during a single period of distributed fields source switching $T=4 \mathrm{~s}$. Description of the technology on how to organize these sinusoidal signals is beyond the scope of this work and will be considered elsewhere.

\subsection{The specific power consumption}

For the same source from Sect. 6.1, the estimation of the power is produced, which is consumed by a distributed source, normalized to the area of the positioning zone.

The investigations have also shown that there are two possible options: single user mode positioning and an unlimited number of users. For a single user only the closest windings are switching on. Otherwise all windings of the distributed source are used in the unlimited number of users mode. For both modes the specific power consumption was found in comparison with $\mathrm{WiFi}$, the most widespread alternative technology.

In the first case, for single user, taking into account the results from the Table 2 for mode $\Delta B=$ Const, the specific power consumption per unit area $P / A$ does not differ from the source with concentrated windings (Blood, 1997):

$\frac{P_{S}}{A}=\frac{R_{i}}{S^{2}} \cdot \frac{1}{T} \int_{0}^{T}\left(\frac{I}{2}\right)^{2}(t) \mathrm{d} t=25 \mathrm{~W} 9 \mathrm{~m}^{2}$, or $<3 \mathrm{~W} \mathrm{~m}^{-2}$.

In the second case, for grid, shown in Fig. 6, we get:

$$
\frac{P_{D}}{A}=\frac{\sum_{i=1}^{9} R_{i}}{16 \cdot S^{2}} \cdot \frac{1}{T} \int_{0}^{T}\left(\frac{I}{2}\right)^{2}(t) \mathrm{d} t \approx 1.5 \mathrm{~W} \mathrm{~m}^{-2} .
$$

It can be seen that the specific powers are approximately equal in both versions $P_{S} \approx P_{D}$, but full powers are proportional to the number of windings $P_{S}=(1 / 9) \times P_{D}$, running simultaneously.

Building regulations give the amount of energy at the level of $60 \mathrm{~W} \mathrm{~m}^{-2}$ (heating plus lighting in middle climatic zone). The proposed distributed source of the positioning fields will add about $3 \mathrm{~W} \mathrm{~m}^{-2}$, i.e., no more than $5 \%$ extra to the existing value. This is acceptable, taking into account all the functional and accuracy benefits of the proposed technology. Thus, the installation of the indoor navigation magnetic system with distributed source of positioning field in modern supermarket building, airport, etc., will not substantially increases the overall power consumption.

\subsection{Evaluation of the positioning errors}

Finally, the above considerations lets one determine the possible accuracy of positioning. For the stationary receiver the measurement error is the sum of the following components:

$B_{\mathrm{c}}-B_{m}=\Delta B_{\mathrm{N}}+\Delta B_{\mathrm{S}}+\Delta B_{\mathrm{F}}$

, where $\Delta B_{\mathrm{N}}$ is the noise of receiver; $\Delta B_{\mathrm{S}}$ is systematic errors of the induction measurement, and $\Delta B_{\mathrm{F}}$ is error of mathematical description of the positioning magnetic field, created by the distributed source. It can be seen that the difference between the measured and calculated values of the induction at any point of positioning zone is defined by both as the measurement error, and the error of description of positioning field.

The random error of measurement can be neglected $\Delta B_{\mathrm{N}} \rightarrow 0$ for a selected duration of a bipolar pulse of the positioning field of $T_{\Pi}=1 \mathrm{~s}$. As investigations have shown, for a coreless winding, the error of the field description is at a level lower than $\Delta B_{\mathrm{F}} / B_{\mathrm{F}} \approx \pm 0.02 \%$. The value of the systematic errors was defined above and can reach a magnitude of $\Delta B_{\mathrm{S}} / B_{\mathrm{S}} \approx 0.6 \%$. The positioning accuracy was determined by the Monte Carlo (Fishman, 1996) method through the transformation of measurement errors to positioning errors: $\sigma_{X} / X \approx \sigma_{B} / B$. Thus, the investigation has shown that if the measurement error is $\sigma_{B} / B \leq \pm 0.6 \%$ then the linear positioning accuracy cannot be worse than $\sigma_{X} / X= \pm 3 \%$, or equivalently $50 \mathrm{~mm}$ at a distance of $1.5 \mathrm{~m}$.

\subsection{Further works}

The next step in advancing the project of active magnetic positioning for indoor navigation should consist of preparing and conducting a full-scale physical experiment, which must demonstrate to potential customers the basic properties of the proposed method. The minimum equipment must comply with Fig. 11.

A further increase in the number of windings allows one to extend the field of application of the experimental results obtained. As part of the planned experiment it the transition 
to autonomous receiver - a smartphone will be made. Before that, the first experimental studies should be conducted on a special receiver similar to the one shown in Fig. 13. The importance to promote of the project is the metrological support of both generation and measurement of positioning field. Initial ideas concerning this issue are formulated and presented in Zhelamskij (2015). As result of this further work the proposed technique will be fully experimentally validated.

\section{Conclusion}

The known methods of indoor navigation have limited accuracy no better than $\pm 1 \mathrm{~m}$ with coverage up to $10 \mathrm{~m}$ and power consumption on the level of $P>1 \mathrm{~W} \mathrm{~m}^{-2}$ for these accuracy and coverage. The best accuracy $(<10 \mathrm{~mm})$ has the method of active magnetic positioning, where the coverage zone is limited by value $R<4 \mathrm{~m}$. However, there is a large influence of EMF and limited update frequency. In order to reduce the impact of EMF and to increase the update frequency, a new method and mathematical model of organization of the positioning field are proposed. This method is built on further improving known magnetic tracking technologies, and allows one, in addition, to reduce the power consumption or to extend the zone of positioning by about $25 \%$.

The method provides a scalable coverage zone due to the synchronized building of elements of the positioning field distributed source. The original way to sync and initial binding (capture) was proposed for autonomous mobile receiver operation.

Thus, assessments show that it is possible to measure the positioning field by using the magnetometer of the modern smartphone, intended to measure the Earth's magnetic field. To do this, a mathematical model is proposed, which takes into account a special source of errors such as the spatial diversity of the receiver sensors. To improve the accuracy of position measurement it may be necessary to individually calibrate the smartphone's magnetometer.

The estimations were made about the values of the power consumption for the distributed source, accuracy of positioning, and update rate of output data. The power usage for the distributed magnetic tracking is minimal among all other known technologies and can be reduced down to $1 \mathrm{~W} \mathrm{~m}^{-2}$. The proposal is aimed at existing mobile devices - smartphones, containing 3-D Earth magnetic field sensor, which could measure the local magnetic field of positioning. The simulation shown that the described method allows for the positioning of a moving object, after increasing the sampling rate of the mobile magnetometer of the smartphone to $2 \mathrm{kHz}$ or higher. In this case the update frequency will increase too.

The sustainability of the new method to the interior is provided by the following circumstances. The ratio between the magnetic pulse duration $(T=0.5 \mathrm{~s})$ and the eddy current decay time constant in the interior $(\tau<1 \mathrm{~ms}) T / \tau \geq 500$ allows one to neglect the influence of electro-conductive interior ele- ments on positioning accuracy (unlike RF technologies). Any dielectric obstacles are transparent for the pulsed magnetic field. The only fatal influence occurs for iron, which is situated between receiver and source (Blood, 1990). Therefore, the floors under the source and remote walls will not affect the positioning accuracy. The modern ferro-concrete has a volume percentage of iron of no more than $5-7 \%$. Therefore, if necessary, accounting for the effects of ferro-concrete barriers can be made by the mapping (Hansen, 1986; Jones, 2002; Zhelamskij, 2015). The body of the mobile user has $\mu \approx 1.00001$ and has therefore no effect on the positioning accuracy, as well as the existence of a permanent magnet in the zone of positioning. The presence of a static magnetic field, for example - residual field created by power facility, during more $6 T$ ( $T$ is the magnetic pulse duration), will also have no effect due to differential measurement in accordance with Eqs. (1)-(3). The dynamic noise is eliminated by averaging filters through the large time of the measurement. Increasing the measurement duration with permanent position of receiver can improve the positioning accuracy to the level $\pm 1 \mathrm{~mm}$ in areas such as underground construction as requested by architects, interior designer, etc.

A comparison of results with other technologies shows that only magnetic tracking provides six coordinates instead three for other technologies. The accuracy of magnetic tracking in indoor navigation is expected as the percentage of the meter that is unachievable for other technologies. The scalable coverage can be provided with the proposed improvement of magnetic tracking opens the way to unlimited zone of positioning, such as the smartphone's network, which is able to cover the entire world.

Acknowledgements. Great thanks Natalia Popova for her faithful support in writing this work. Also, the author is deeply grateful to old Swiss friend and colleague Alexander Anghel from PSI for assistance in accurate scientific translation of articles into English.

Edited by: I. Bársony

Reviewed by: three anonymous referees

\section{References}

Altman, A. C., Ephrathm, Y., and Govari, A.: Position tracking using quasi-dc magnetic fields, United States patent US 2039315, 25 March 2009.

Asahi - Kasei: AK8975/AK8975 3-axis Electronic Compass, http: //www.asahi-kasei.co.jp/asahi/en/, last access: 14 October 2016.

Blood, E.: Device for quantitative measuring the relative position and orientation of two bodies in the presence of metals utilizing direct current magnetic fields, Ascension, United States patent US 4945305, 1990.

Blood, E.: Device for measuring position and orientation using nondipole magnet DC fields, Ascension, United States patent US 5600330, 1997. 
Bosh: 9 axis motion tracking, https://www.bosch-sensortec.com/ bst/products/all_products/bmx055, last access: 26 October 2016.

Chao, H., Das Saumitra, M., Gupta, R., Khorashadi, B., Sridhara, V., and Pakzad, P.: Adaptive updating of indoor navigation assistance data for use by a mobile device, United States patent US 9081079 B2, 7 November 2013.

Daly, D., Melia, T., and Baldwin, G.: Concrete embedded RFID for Way - point positioning. Processing of International Conference on Indoor navigation (IPIN), ETH Zurich, Switzerland, 2010.

Dardari, D., Luise, M., and Falletiis, E.: Satellite and terrestrial radio positioning techniques, a signal processing perspective, Elseiver Ltd, Oxford, UK, 2012.

Fishman, G.: Monte Carlo: concepts, algorithms, and applications, Springer, ISBN: 0-387-94527-X, 1996.

Fuller, R. and Malkos, S.: Indoor/Outdoor Differentiation Using Radio Frequency Transmitters, United States patent 2680039 A1, 1 January 2014.

Hansen, P.: Magnetic position and orientation measurement system, United States patent US 4622644, 1986.

Invensense: 9 axis motion tracking, http://www.invensense.com/ products/motion-tracking/9-axis/, last access: 14 October 2016.

IPIN: Proceedings of the 2015 International Conference on Indoor Positioning and Indoor Navigation. University of Calgary, 643 pp., ISBN: 1467384038, 9781467384032, 2015.

Jones, H.: Method and apparatus for determining electromagnetic field characteristics within a volume, Polhemus, United States patent US 6377041, 2002.

Mandal, A., Lopes, C. V., Givardis, T., Haghighat, A., Jurdak, R., and Baldi, P.: Beep: 3D Indoor positioning using audible sound, Processing of the Consumer Communications and Networking Conference (CCNC 2005), IEEE Xplore, 2005.

Mautz, R.: Indoor Positioning Technologies, Habilitation thesis, Institute of Geodesy and Photogrammetry, ETH Zurich, 2012.
Melzer, J. and Moffit, K.: Head mounted displays, Designing for the User, McGraw-Hill Companies, Inc., 1997.

Microsoft: The optical scanning system, https://en.wikipedia.org/ wiki/Kinect, last access: 14 October 2016.

Navizon: Cell-ID positioning solution, https://navizon.com, last access: 26 October 2016.

Pirkl, G. and Lukowicz, P.: Resonant magnetic Coupling Indoor Localization System, Proceeding of "UbiComp'13", Zurich, Switzerland, 2013.

Scully, J. and Schneider, M.: DC magnetic-based position and orientation monitoring system for tracking medical instruments, United States patent US 8942780 B2, 27 January 2015.

Zhelamskij, M.: A method for determining the position and orientation of movable object, Russia Federation patent RF 2413957, 2011.

Zhelamskij, M.: Features of the Measurement of a Local Positioning Magnetic Field at Short Distances, Meas. Tech., 57, 1032 1040, 2014a.

Zhelamskij, M.: Features of the Construction of a Positioning Field for Local Navigation in Enclosed Spaces, Meas. Tech., 57, 791799, 2014b.

Zhelamskij, M.: The positioning of mobile objects in local magnetic field, Saarbrucken: Palmarium Academic Publishing, ISBN: 978-3-659-60217-7, 340 pp., 2015 (in Russian).

Zhelamskij, M.: The First domestic magnetic tracker, Sensors \& Systems, 1, 2-7, 2009 (in Russian).

White, B. O., Kim, J. D., and Lee, S. E.: Method and apparatus for tracking a global navigation satellite system within the constraints of an indoor facility, United States patent US 9121937 B2, 1 September 2015. 